
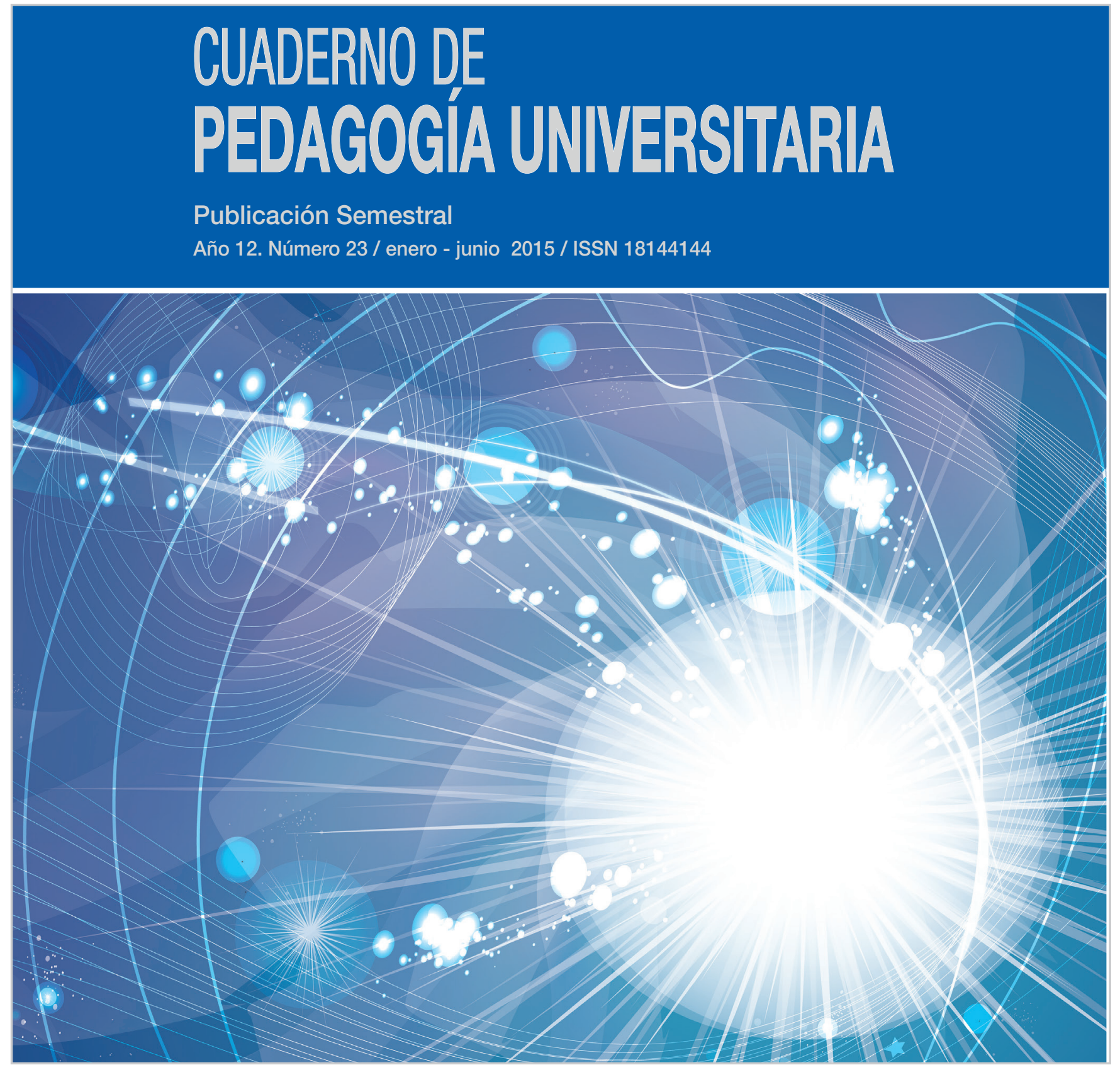

En esta sección compartimos las opiniones que nos envían nuestros lectores sobre los artículos publicados en el último ejemplar del Cuaderno de Pedagogía Universitaria: No. 23 / enero- junio 2015

\title{
Manuales de Procedimientos: contribuyendo con el proceso de Enseñanza-Aprendizaje en Administración Hotelera
}

En este artículo se pude apreciar cómo la profesora Frances Grullón desempeña su función docente apegada al nuevo modelo educativo de nuestra universidad para renovar una práctica de pasantías en Administración Hotelera que estaba desconectada del proceso Enseñanza-Aprendizaje. Me pareció muy acertado iniciar este trabajo con un diagnóstico 
para saber específicamente qué debía ser mejorado para luego formular un plan de acción. Sumar al estudiante en cada etapa del proceso iniciando desde la solicitud de la pasantía y hacerlo partícipe de los intercambios, empodera al estudiante de su proceso de aprendizaje, creando una mayor vinculación en esta importante etapa estudiantil. De igual manera, la rúbrica de evaluación y la inclusión de diarios reflexivos en las actividades asegura el aumento de la calidad de los criterios de evaluación y sus resultados. Por último, la utilización de la Plataforma Virtual de Aprendizaje para dar seguimiento al proceso acompañado de visitas y llamadas muestran al estudiante el camino hacia su formación integral como futuro profesional.

Felicito a la maestra por todo el trabajo que significó este manual de procedimientos para convertirlo en una herramienta con la que se mejore la calidad de las pasantías especificas brindando a la vez resultados medibles y tangibles que fortalecen el vínculo de la formación académica con el mundo laboral.

Definitivamente, se utilizan verdaderos instrumentos pedagógicos para el aprendizaje profundo del estudiante en cada proceso y por supuesto que todo este arduo trabajo tiene como resultado un aprendizaje significativo, mejor actitud estudiantil y sentido de responsabilidad sobre los procesos educativos augurando el éxito de todos los esfuerzos que constantemente hacen la PUCMM y sus maestros para actuar apegados a los más exigentes estándares de la educación universitaria.

Prof. Samuel Cornielle Profesor de Departamento Lingüística Aplicada Campus Santo Tomás de Aquino

\section{Implementación del proceso de producción escrita para el mejoramiento de la redacción de textos expositivos en Administración Hotelera}

El artículo "Implementación del proceso de producción escrita para el mejoramiento de la redacción de textos expositivos en Administración Hotelera" escrito por la profesora Kenia Rodríguez demuestra de una manera sencilla la forma en que se puede sumergir al estudiante en situaciones problemáticas en su campo de acción laboral y luego a partir de su vivencia, escribir textos expositivos. Es una estrategia que podría perfectamente extrapolarse a otros contextos académicos tomando en cuenta las problemáticas propias de la disciplina en que se pretende aplicar. Inducir a los estudiantes en problemáticas reales los coloca frente a lo que será su accionar, donde deben aprender a buscar las soluciones pertinentes.
Por otro lado, esta estrategia consigue que los estudiantes produzcan textos expositivos apegados a los criterios exigidos por los expertos en la disciplina en que se esté implementando, lo cual fortalecerá sus competencias de pensamiento crítico, así como la mejoría en su comprensión escrita. En fin, considero el artículo como una motivación para un docente de cualquier disciplina que desee que sus alumnos escriban de manera correcta y analicen los efectos de una problemática de su área para tomar las decisiones pertinentes de manera objetiva y apropiada al contexto.

Minerva González Germosén Profesora del Departamento de Español Campus Santo Tomás de Aquino

Externo mis felicitaciones a la profesora Kenia Rodríguez, reconociendo sus méritos al implementar un estudio de investigación-acción. Luego de leer y participar en la presentación del estudio, pude valorar los altos beneficios tales como implementar estrategias de enseñanza, utilizar la escritura de ensayos reflexivos como recurso de aprendizaje, así como el empleo de una guía que orienta al estudiantado en sus directrices de redacción, y el empleo de una rúbrica de evaluación.

A pesar de considerar que la escritura es un desafío para los aprendices, su uso permite desarrollar la destreza de elaborar textos con apropiada fluidez lo cual es favorable para el perfil del egresado. La elaboración de ensayos, informes o cualquier tipo de texto, requiere una mirada sistémica del tema en cuestión y el estudiante debe realizar una investigación exhaustiva sobre el tópico a tratar, plantear los distintos puntos de vistas, así como su percepción personal.

Además, la elaboración de la guía, permite conducir al estudiante sobre los aspectos fundamentales en los cuales debe investigar y redactar. A su vez calibra la evaluación por parte de la rúbrica e iguala la producción escrita frente a los demás estudiantes. Fue de alto valor esta investigación-acción al observar las mejorías que se muestran en las gráficas. A su vez, los estudiantes expresaron empatía y motivación mostrando una alta satisfacción. Esta estrategia ofrece la oportunidad de que otros docentes también puedan emplear este recurso para la mejoría de nuestros estudiantes.

$$
\begin{array}{r}
\text { Adien Lugo } \\
\text { Profesor del Departamento de Medicina } \\
\text { Campus de Santiago }
\end{array}
$$

¿Quién es mi cliente, como es él, de dónde viene, a qué viene, volverá? ¿Por qué vino a mí, qué le puedo ofrecer, cuánto le puedo ofrecer? 
Un buen administrador hotelero debe tener una versatilidad única para competir en el mundo de hoy con resultados favorables, pues realmente, las exigencias de nuestros clientes y de los potenciales son sumamente complejas, con el agregado de las limitaciones socioculturales de nuestro medio. Es aquí, donde la buena escritura viene en auxilio de esta profesión: Acorta distancias, reduce tiempo, es oportuna, deja constancia y puede lograr un cambio de actitud en el receptor. Ahora entiendo mejor la preocupación de la profesora Rodríguez que le impulsó a realizar esta investigación para acercarse lo más posible al perfil del egresado de ADH que necesita nuestro país.

Los hallazgos de la investigación validan el proceso para mejorar el desenvolvimiento en la escritura de nuestros estudiantes, lo cual se constituye en una ganancia significativa para nuestro Modelo Educativo: lograr un estudiante con una formación más amplia (la redacción sería un extra); el proceso de enseñanza-aprendizaje se fortalece considerablemente ya que para escribir sobre un determinado tema se requiere un mayor dominio del mismo y cierto grado de criticidad, esto afianza y fragua el aprendizaje. $Y$ como arte, la escritura puede producir en el individuo una satisfacción en el quehacer mismo que lo vuelve más ameno y gratificante. Con el desarrollo de las competencias de la buena escritura definitivamente damos un golpe fulminante al copiar y pegar.

José Aridio De los Santos Departamento de Educación Física Campus de Santiago

\section{Valoración de las competencias implicadas en la formación del arquitecto en la Pontificia Universidad Católica madre y Maestra}

Este articulo realiza una interesante revisión y análisis de la relación entre las competencias declaradas en el proyecto TUNINGAL y el lugar que a estas dan los diferentes actores de la comunidad universitaria en los departamentos de Arquitectura de ambos campus. Profundiza en el proceso de cambio del currículo hacia un modelo por competencias, donde se declara importante reconocer el peso de cada una de estas en la malla curricular y su influencia en la formación disciplinar.

El aporte de esta investigación radica en arrojar luz sobre lo que se considera un campo disciplinar muy desdibujado, pues la enseñanza de la Arquitectura ha sido planteada por autores como un escenario misterioso y poco explicitado.
Es importante anotar que la metodología llevada a cabo por los investigadores sistematiza una forma de hacer investigación-acción en un ambiente de formación disciplinar muy peculiar y especial.

Se presenta el desglose de la relación de los niveles de dominio de las competencias y su implicación en las asignaturas de la malla curricular lo que ayuda en aclarar los aspectos que muchas veces quedan sueltos por no estar explícitamente declarados.

Felicito al equipo de investigadores por la iniciativa de publicar y con esto compartir sus hallazgos con la comunidad académica, lo cual abre el camino para que la carrera de Arquitectura siga caminando sobre caminos certeros.

\section{Orisell Medina Lagrange \\ Profesora del Departamento de Arquitectura Campus Santo Tomás de Aquino}

Este artículo me ha llevado a la reflexión de cómo todas las carreras de nuestra Universidad irán entrando en procesos de rediseño para irlas adaptando a nuestro Modelo Educativo. A partir de la lectura y de lo socializado en la Peña Pedagógica, he comprendido mejor que debemos ir pensando, cómo adaptar nuestro quehacer académico a este modelo de enseñanza por competencias y cuáles son las competencias genéricas y específicas que deben conformar los planes de estudio de cada una de nuestras carreras, incluso antes de que toquen a nuestra puerta y nos impulsen a un proceso de rediseño. Sin dudas, este debe iniciarse antes con un proceso de convencimiento, desde abajo.

El Proyecto Tuning América Latina (TUNINGAL), utilizado por los autores como marco para el artículo, también analizó la carrera de Derecho. Este proyecto constituirá un referente importante para cuando se pretenda iniciar un proceso de rediseño de la carrera en la PUCMM.

Dariel Suárez

Profesor del Departamento de Ciencias Jurídicas Campus de Santiago

Opinión de artículo sobre "Modos de organización de los textos expositivos y su relación con la comprensión escrita" por Lic. Ramira Disla

Ciertamente, el procesamiento de la información de aquello que se lee y se comprende es un holismo de gran relevancia en las distintas asignaturas pues 
promueve el logro de competencias esenciales para el desarrollo cognitivo del individuo, aunque lamentablemente, el profesorado no acostumbra a incentivarlo, siendo esto una deficiencia de la docencia.

El proceso cognitivo que engloba la comprensión escrita conlleva interpretación, el razonamiento, la memoria, el lenguaje, la toma de decisiones, la resolución de problemas, inclusive los sentimientos, los cuales son elementos vinculados entre sí que deben potenciarse en toda institución educativa como parte de la formación del individuo.

Dra. Daliana Gómez Facultad de Estomatología Campus de Santiago

Entre los artículos incluidos en el número 23 del Cuaderno de Pedagogía Universitaria, llama mi atención el trabajo publicado por la profesora Ramira Disla, basado en los resultados de una investigación llevada a cabo con estudiantes de Filosofía.

La profesora Disla toca un punto muy sensible que atañe a la calidad del proceso de enseñanza aprendizaje, pues al analizar los resultados de su investigación acerca de los modos de organización que dificultan la comprensión escrita, se demostró que persisten grandes deficiencias entre los estudiantes para comprender lo que leen.

Debemos prestar atención a las recomendaciones de utilizar el resumen como estrategia para mejorar la interpretación, así como a aplicación de las diversas metodologías recomendadas para una mejor comprensión de los textos expositivos, lo cual fortalecería el proceso de enseñanza aprendizaje.

La autora ha sido muy puntual al señalar que la formación de sujetos capaces de comprender los textos que leen es fundamental para el logro de aprendizajes para la vida.

Ariosto Díaz
Profesor del Departamento de Estomatología
Campus de Santiago

Comentario sobre Pasos y huellas: entrevista a la profesora Rosa Noyola

La entrevista donde se reseña la vida académica de la Profesora Rosa Noyola fue un elemento valioso en la publicación del Cuaderno de Pedagogía Universitaria.
Es un testimonio muy motivador el hecho de conocer las experiencias de una persona que ha ocupado distintos cargos en la PUCMM, y que posee un caminar que va dejando huellas con su ejemplo y dedicación tesonera a la construcción de la historia de la Universidad y la incidencia de su trabajo repercute en la vida de tantos egresados, y en todos aquellos que laboran junto a ella.

Doña Rosa es un gran ejemplo de entrega y dedicación en lo científico, humano y profesional, a través de un mismo hilo conductor que ha sido la rectitud y seriedad de propósito con las cuales se ha entregado, por lo cual la reconocemos como una persona que ha dedicado sus mejores esfuerzos para la consolidación de la calidad académica de la PUCMM.

Enhorabuena para la profesora Rosa Noyola y a nuestra revista para que siga delante como vocera de la comunidad académica.

Prof. Inmaculada Torres

Departamento de Educación

Campus Santo Tomás de Aquino

\section{Reseña del libro Tutoría Universitaria Inclusiva. Guía de buenas prácticas}

Resulta extremadamente interesante la síntesis que hace la autora Cristina Amiama sobre este libro de la autoría de Pedro R. Alvarez Pérez, el cual, como ella explica, fue escrito básicamente para guiar el manejo de estudiantes con necesidades educativas especiales, por lo cual, el contenido del mismo puede aplicarse de manera efectiva a nuestra institución y a sus propias particularidades y a raíz de su análisis podemos identificar áreas para desarrollar o mejorar en la PUCMM.

Me gustaría resaltar dos aspectos sobre los cuales esta lectura me ha hecho meditar. El primer aspecto fue el de los estudiantes que se encuentran A Prueba Académica y su necesidad de apoyo para lograr salir de su condición. En el caso de ADH, ADM y otras carreras de la Universidad, se ha diseñado un plan de orientación en cuanto a las asignaturas a tomar y la combinación de clases adecuada para el logro de esta meta. Sin embargo, leyendo este artículo se me ocurre que podemos diseñar un plan a través del cual se integre además alos profesores, quienes podrían asumir un compromiso junto a la dirección para, a través de "tutorías de supervisión del trabajo académico", ayudar de manera más efectiva a este tipo de estudiantes. 
El segundo aspecto de reflexión ha sido la educación inclusiva, la cual se refiere a los estudiantes con necesidades especiales. Esta es una realidad en nuestra sociedad, como lo es en el mundo. A nivel de nuestra universidad existe la urgencia de diseñar un plan para acoger de manera efectiva estos estudiantes.

Para lograrlo, es necesaria la formación de todo el personal universitario con respecto a la relación con personas con discapacidad, especialmente a los docentes y personal administrativo. Es también muy importante disponer de personal especializado que apoye el proceso formativo de este tipo de estudiantes, sobre todo en los casos de discapacidad visual o auditiva.

Entiendo que temas como éste deben encaminarnos hacia un diálogo institucional que nos lleve a diseñar soluciones creativas y efectivas a corto plazo.

\section{Lic. Miriam Laureano, MBA \\ Profesora Instructora ADH Campus Santo Tomás de Aquino}

La profesora Amiama hace una reseña que nos motiva a buscar el libro para profundizar sobre lo que plantea Álvarez Pérez. Asimismo, expresa claramente la necesidad de la colaboración entre profesores, la formación en atención a la diversidad y un interés real del docente de tomar en cuenta el enriquecimiento que supone la valoración de las diferencias de los estudiantes. Se propone dar una respuesta a la diversidad, no solo desde las discapacidades, sino que invita a una nueva mirada a diferentes temas de la persona del estudiante.

El artículo tiene una particular importancia en el contexto en que se desenvuelve el profesor universitario, donde normalmente se espera que el estudiantado tenga desarrollada la mayoría de las habilidades y a partir de su egreso como bachiller. La reseña que hace la profesora Amiama destaca uno de los aspectos que alude Álvarez Pérez y nos lleva a reflexionar sobre la importancia de tomar en cuenta las necesidades específicas de apoyo educativo (NEAE) y asumirlo "como un reto que nos implica a todos (...)" (Citado por Amiama).

Lic. Ysa Bisonó

Profesora de Departamento de Orientación Campus Santo Tomás de Aquino

\section{Retos formativos y pedagógicos del doctorado en el siglo XX}

El interesante texto del Dr. Enrique Sánchez Costa sobre la formación de doctores me lleva a completar

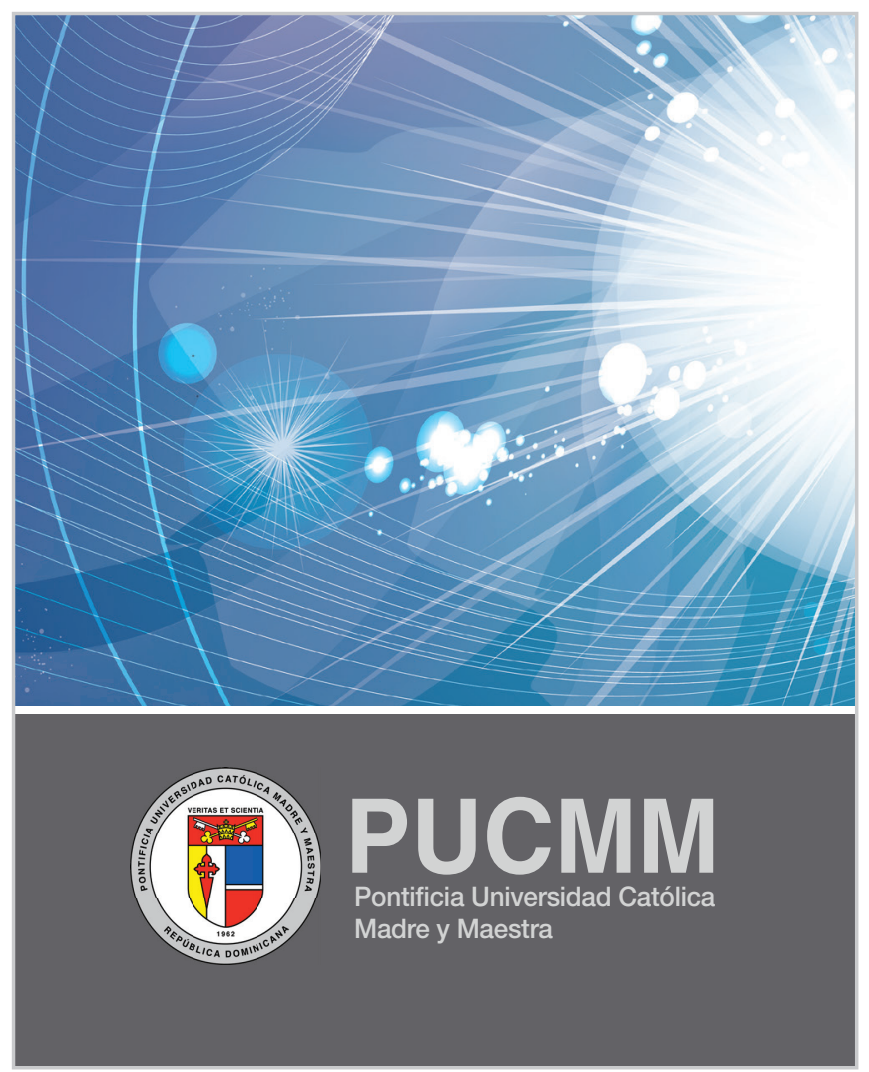

un ejemplo que él señala como malogrado con la experiencia de China en su programa doctoral. En mi obra, China, ¿el futuro de la globalización? (1998) presento la primera de las cuatro modernizaciones que inició el vicepresidente y hombre fuerte del post-maoísmo, Deng Tsiao-Ping, que consistió en restaurar el criterio de verdad científica por encima de la verdad ideológica, lo que significó iniciar una serie de universidades tecnológicas, doce en total, para comenzar desde cero la formación "rigurosa" y dejando a la deriva al resto de universidades que se adaptaran al nuevo criterio de verdad.

El ejemplo del Dr. Sánchez Costa puede llevarnos a pensar en el estancamiento en la educación doctoral, pero el dato después del primer bache que consigna, la medición del avance científico lo mide la inversión en investigación y desarrollo, que China se sitúa como segunda en el mundo detrás de Estados Unidos de América. Como señala Batelle.org, China en 2011 tuvo $1.4 \%$ de su PIB como gasto en investigación y desarrollo, sólo por detrás de Estados Unidos de América, que dedicó en el mismo año 2.7\%. Considero importante señalar la historia completa. Por lo demás, muy interesante la reflexión del Dr. Sánchez Costa.

Prof. Miguel Sang Ben Profesor de Economía y Educación Campus de Santiago 